\title{
Detection of Faults in Implanted Cardiac Pacemakers
}

\author{
G. D. GREEN, W. FORBES, W. H. BAIN, G. B. SHAW, AND A. C. F. KENMURE \\ From the Department of Clinical Physics and Bio-Engineering, Western Regional Hospital Board, 11 West Graham \\ Street, Glasgow C.4, and the Royal and Western Infirmaries and Southern General Hospital, Glasgow
}

Since 1958, totally implantable pacemakers have been used to stimulate the hearts of patients suffering from chronic heart block. The evidence that such patients have a reduced mortality rate in comparison with unpaced patients (Sowton, 1967) and the ability to return to a normal life have meant that pacemaker implantation for heart block is now accepted practice. There have been increasing numbers of reports on the improved reliability of such pacemakers (Parsonnet, Gilbert, and Zucker, 1966; Forbes et al., 1968; Sowton, 1968), but nevertheless failures do occur and it is most important that continued research into methods of anticipating and diagnosing such failures should continue. Sowton (1968) has emphasized the need for followup arrangements to be carried out at specially equipped clinics. At the same time, it should be appreciated that there is as yet no single test or method which will satisfactorily diagnose all possible faults (Dekker, Büller, and Schuilenburg, 1965). We have made a preliminary announcement of a method of testing the integrity of lead systems (Green et al., 1968) in which an analysis is made of the vector produced in the frontal plane by the pacemaker signal obtained using an oscilloscope and standard surface electrocardiographic electrodes. This paper reports the results of our experience in using this method in three Glasgow pacemaker clinics.

As seen in an electrocardiogram, the pacemaker signal has the appearance of a sharp deflection immediately before the QRS complex, usually negative in leads I and II with a bipolar catheter and either positive or negative in lead III (Fig. 1). By excluding the electrocardiogram signal and examining the pacemaker signal on an oscilloscope (preferably a storage oscilloscope), the characteristics of the pacemaker signals can be seen in more detail (Fig. 1). The pacemaker signal is a "square-wave" pulse of electricity, two milliseconds or less in dura-

Received April 24, 1969. tion, a few millivolts in amplitude, and its parameters can be measured with a suitable oscilloscope, as described by Knuckey, McDonald, and Sloman (1965). It is possible to draw the frontal plane vector of the pacemaker pulses from the amplitude measurements by simple vectorial addition of the signals obtained from any 2 of the 3 standard body
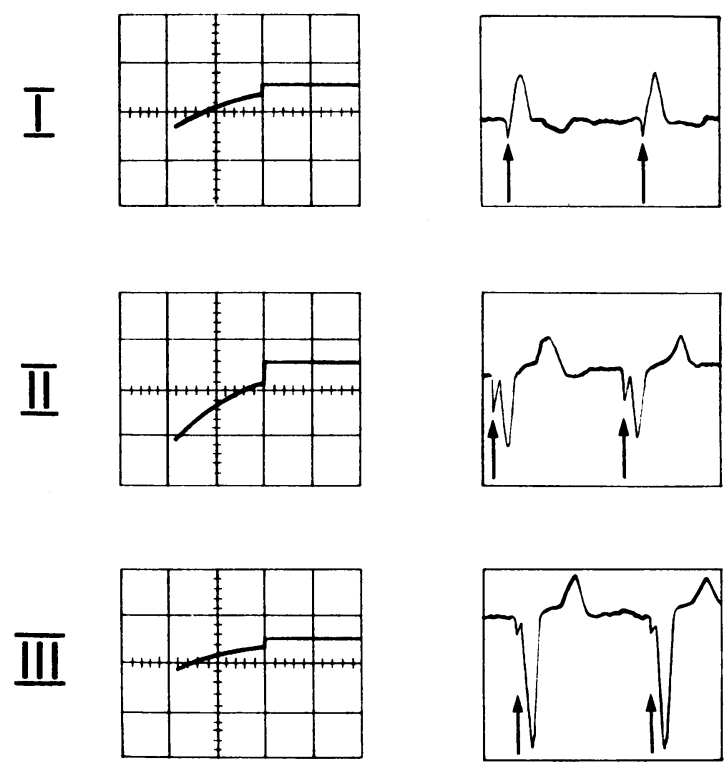

FIG. 1.-Typical electrocardiograms and corresponding oscilloscopic records of pacemaker signals (leads I, II, and III). Arrows on electrocardiograms show pacemaker pulses.

electrocardiograph surface leads. Using this simple technique, the direction and magnitude of the frontal plane pacemaker vector can be readily examined. The former indicates the resultant direction of current flow, which in the normal pacemaker system will be from the negative (distal) electrode to the positive (proximal) electrode as they lie in the 707 
direction between 8 and 11 o'clock. Consecutive frontal plane pacemaker vectors obtained over a period of time from the same patient may be spread over an angle such as $25^{\circ}$ (Fig. 2), but with present techniques smaller angles of spread are more common. It is also hoped to relate consecutive decreases in magnitude of the frontal plane pacemaker vector in the same patient with battery depletion. the negative lead broke near the point of entry of the catheter into the external jugular vein.

Break in Insulation on Positive Lead. The heart may again continue to be paced when the insulation on the positive lead breaks. In these circumstances electron current flows from the distal negative electrode at the tip of the catheter to the proximal

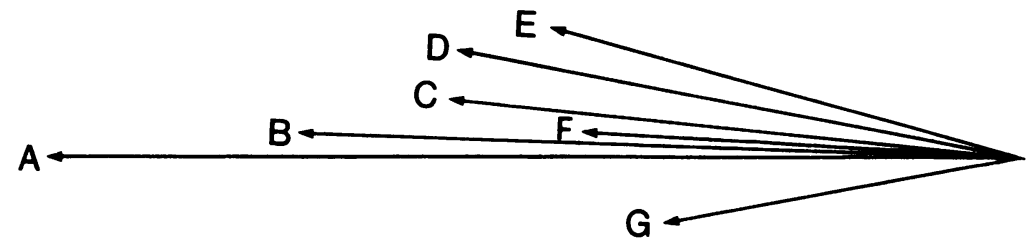

FIG. 2.-Frontal plane pacemaker vectors obtained from the same patient. A, B, C, etc. taken at successive three-monthly intervals.

\section{Material AND METhods}

Between June 1962 and December 1968, 155 pacemakers have been implanted in 89 patients in the Western Region of Scotland. Since October 1964, the Medtronic bipolar endocardial axillary (fixed rate) pacemaker has been preferred in this Region and most of the tests described below have been carried out on this model, with the generator placed in the right axillary region. Excluding a number of deaths not necessarily due to pacemaker failure and also reoperations carried out for reasons other than electromechanical failure, 11 generators (and possibly 2 more unobtainable after death) and 7 catheters have failed since October 1964. These patients are seen at 3 special clinics in the City of Glasgow (Royal Infirmary, Western Infirmary, and Southern General Hospital) at intervals of 1 or 2 months. After interview and examination a conventional electrocardiogram is recorded; with the electrodes still in place the standard lead recordings are then fed into a storage oscilloscope. We have used the technique of frontal plane vector analysis of pacemaker signals in our follow-up clinics since March 1966 and now have over 350 records on 76 patients.

\section{RESULTS}

Break in Insulation on Negative Lead. If the insulation on the negative lead breaks, the heart may continue to be paced because the current can still flow in 2 paths both of which could stimulate contraction; 1 path is between the bipolar electrodes and the other is between the point in the negative lead where the insulation has broken and the positive electrode. In these circumstances the resultant frontal plane pacemaker vector is rotated through a large angle anticlockwise (Fig. 3). This has been seen in 4 patients, 1 of whom is still being paced satisfactorily. In 2 of these cases the insulation on

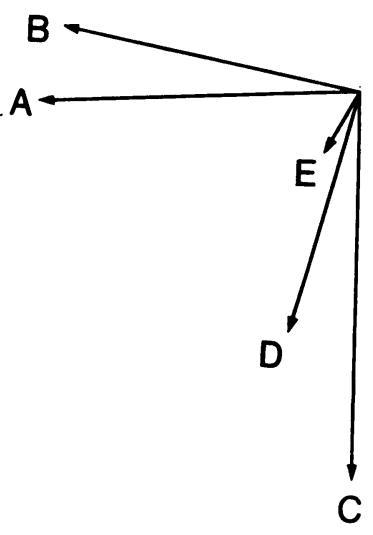

Fig. 3.-Anticlockwise rotation of pacemaker frontal plane vector in patient shown to have break in insulation on negative lead. Vectors $A$ and $B$, before break in insulation. Vectors $\mathrm{C}, \mathrm{D}$, and $\mathrm{E}$, after break in insulation.

positive electrode and also from the distal negative electrode to the point in the positive lead where the break has occurred, so that the resultant frontal plane pacemaker vector is roughly in the same direction as the original vector. In practice, the resultant frontal plane pacemaker vector is rotated through a small angle clockwise with respect to the direction of the original vector. With the generator implanted in the right axilla there may be some diffculty in observing such a small clockwise rotation. We have not so far encountered a break in the positive lead insulation. However, this was suspected in a recent case and effectively the same fault was found at reoperation when it was observed that the insulating screw on the positive terminal was missing. After another insulating screw had been fitted 
and the second conducting path thereby removed, there was the anticipated change in the position of the frontal plane pacemaker vector. This is illustrated in Fig. 4.

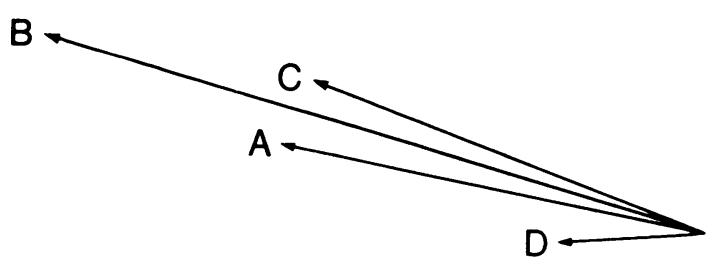

Fig. 4.-Clockwise rotation of frontal plane pacemaker vector. $D$, normal vector, pacemaker intact. A, B, C, positive insulating screw missing.

Break in Conducting Leads. If the conducting lead itself breaks, but its insulation remains intact, the pacemaker pulses may still reach the heart if the ends of the broken conducting lead are still touching each other. However, because of the high resistance introduced into the conducting lead the size of the pulse at the catheter electrodes will be much smaller. Fig. 5 shows the change in the

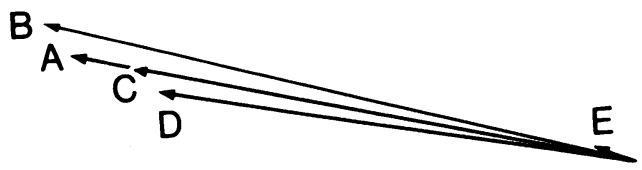

Fig. 5.-Conspicuous change in effective output of pacemaker after break in conducting lead is shown by sudden reduction in vector magnitude ( $E$ taken on May 5,1968 ) compared with previous vectors (A-D taken between May 12, 1967 and January 1, 1968).

length of the frontal plane pacemaker vector when such a lead break occurs and in this instance at operation a break was found in the negative conducting lead, the insulation being intact. Two other similar cases have occurred in our series and the breaks have been found in that part of the lead between the positive and negative electrodes.

We have no experience of a break occurring in a positive conducting lead but the arguments given above still apply.

\section{Discussion}

The technique described above has proved useful in the detection of faults associated with gross changes in either the direction or magnitude of the frontal pacemaker vector in our series of endocardial fixed rate pacemakers, and it has now been extended to include endocardial demand (QRS-blocking) pacemakers as well as epicardial pacemakers.

In our earlier studies we at first failed to appreciate the full effect of respiration on the direction of the frontal plane pacemaker vector, thereby running the risk of wrongly interpreting changes in direction. However, this can be significantly reduced by taking measurements only in full inspiration, which is now our practice.

On a practical note, we have already mentioned difficulties in interpretation due to the position of the generator in the right axilla when a break occurs in the positive lead insulation. If, however, the generator is implanted in the patient's left axilla such a break would be associated with a greatly increased clockwise rotation of the vector and diagnosis of such a fault would be made more certain. This may constitute a case for left axillary implantation.

The relation between the magnitude of the frontal plane pacemaker vector and the output of the pacemaker is affected by (a) catheter movements within the heart, (b) catheter movements caused by respiration, and (c) catheter movements arising from cardiac activity.

With regard to factor (a), necropsy evidence suggests that the catheter becomes fixed by the formation of a fibrous sleeve. The effects of respiration (b) can again be reduced by taking measurements in full inspiration. The effect of the third factor (c) is small.

All the effects of catheter movements should be eliminated by three-dimensional vectorcardiography. This is at present being studied in conjunction with the University Department of Medical Cardiology, Royal Infirmary, Glasgow.

It is worth noting that only one of the faults we have mentioned may result in immediate failure to pace the patient. These faults cannot be detected by clinical examination alone and some form of simple electronic testing is essential at a pacemaker clinic. As our knowledge, experience, and confidence in the technique described have increased, there are indications that it may be of use in battery depletion studies, particularly at the later stages of implantation of a pacemaker.

\section{SUMMARY}

A simple technique has been described which enables the following pacemaker faults to be detected from a knowledge of the position and magnitude of the pacemaker frontal plane vector. Breaks in the insulation of either lead-the conducting leads themselves remaining intact. Breaks in either 
of the conducting leads, the insulation remaining intact and the generator rate remaining unchanged. Errors at operation: if an abnormal frontal plane pacemaker vector is obtained immediately after an implant has taken place this should be studied and the possibility of error at operation considered.

The technique may prove to be of use in battery depletion studies at the later stages after implantation.

We wish to thank the many physicians, cardiologists, and cardiac surgeons in the Western Region of Scotland who have referred cases to the clinics at the Royal and Western Infirmaries and Southern General Hospital in Glasgow. We also gratefully acknowledge assistance given by Dr. J. M. A. Lenihan, Regional Physicist, and other colleagues in the Regional Department of Clinical Physics and Bio-engineering, Western Regional Hospital Board, Glasgow.

\section{REFERENCES}

Dekker, E., Büller, J., and Schuilenburg, R. M. (1965). Aids to electrical diagnosis of pacemaker failure. Amer. Heart f., 70, 739.

Forbes, W., Green, G. D., Shaw, G. B., and Bain, W. (1968). Long-term transvenous cardiac pacing with ChardackGreatbatch (Medtronic) pacemaker. Brit. med. f., 2, 13.

Green, G. D., Forbes, W., Bain, W. H., and Shaw, G. B. (1968). Detecting break in insulation in negative pacemaker lead. Brit. med. F., 4, 645.

Knuckey, L., McDonald, R., and Sloman, G. (1965). A method of testing implanted cardiac pacemakers. Brit. Heart F., 27, 483.

Parsonnet, V., Gilbert, L., and Zucker, I. R. (1966). Permanent pacemaker insertion. A five-year appraisal. Ann. thorac. Surg., 2, 561.

Sowton, E. (1967). Cardiac pacemakers and pacing. Mod. Conc. cardiov. Dis., 36, 31.

- (1968). Results of a British survey on artificial cardiac pacemakers. Brit. med. $\mathcal{F}$., $2,11$. 